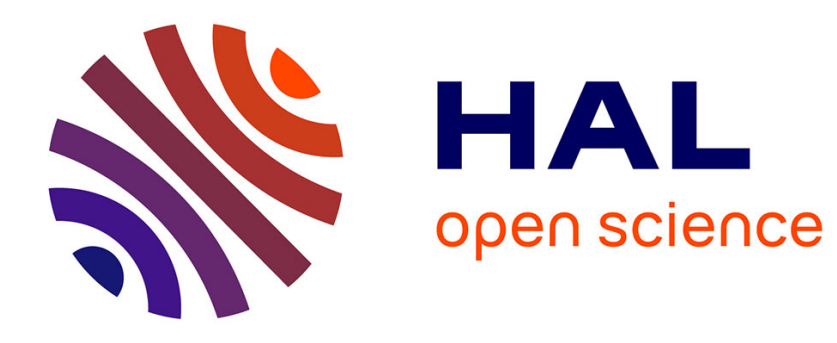

\title{
On the decay of turbulence in plane Couette flow
}

Paul Manneville

\section{To cite this version:}

Paul Manneville. On the decay of turbulence in plane Couette flow. Fluid Dynamics Research, 2011, 43 (6), pp.065501. 10.1088/0169-5983/43/6/065501 . hal-01025564

\section{HAL Id: hal-01025564 \\ https://hal-polytechnique.archives-ouvertes.fr/hal-01025564}

Submitted on 18 Jul 2014

HAL is a multi-disciplinary open access archive for the deposit and dissemination of scientific research documents, whether they are published or not. The documents may come from teaching and research institutions in France or abroad, or from public or private research centers.
L'archive ouverte pluridisciplinaire HAL, est destinée au dépôt et à la diffusion de documents scientifiques de niveau recherche, publiés ou non, émanant des établissements d'enseignement et de recherche français ou étrangers, des laboratoires publics ou privés. 
On the decay of turbulence in plane Couette flow

This content has been downloaded from IOPscience. Please scroll down to see the full text.

View the table of contents for this issue, or go to the journal homepage for more

Download details:

IP Address: 129.104.29.1

This content was downloaded on 18/07/2014 at 07:58

Please note that terms and conditions apply. 


\title{
On the decay of turbulence in plane Couette flow
}

\author{
Paul Manneville \\ Hydrodynamics Laboratory, École Polytechnique, Palaiseau 91128, France \\ E-mail: paul.manneville@polytechnique.edu
}

Received 25 January 2011, in final form 10 August 2011

Published 21 October 2011

Online at stacks.iop.org/FDR/43/065501

Communicated by M Asai

\begin{abstract}
Upon decreasing the Reynolds number, plane Couette flow first forms alternately turbulent and laminar oblique bands out of featureless turbulence below some upper threshold $R e_{\mathrm{t}}$. These bands exist down to a global stability threshold $R e_{\mathrm{g}}$ below which laminar flow ultimately prevails. We study the fragmentation and decay of these bands in systems that are extended enough for several bands to exist. We use direct numerical simulations appropriately tailored to deal with such large systems during long enough durations. We point out a two-stage process involving the rupture of a band and then its slow shrinking. Previous interpretations of turbulence decay in wall-bounded flows within the chaotic transient or spatiotemporal intermittency paradigms are discussed.
\end{abstract}

(Some figures in this article are in colour only in the electronic version)

\section{Introduction}

The transition to turbulence in wall-bounded flow is a long-standing problem of great theoretical and practical importance. Specific difficulties arise from its hysteretic character linked to the fact that the nontrivial turbulent regime stands at a distance from the laminar regime and may coexist with it. On general grounds, laminar flow prevails as the unique permanent regime, i.e. in the long-time limit and whatever the initial state, below some value $R e_{\mathrm{g}}$ of the Reynolds number $R e$ called the global stability threshold. On the other hand, a linear instability threshold $R e_{\mathrm{c}}$ can usually be obtained from standard stability analysis, beyond which the base profile is unconditionally unstable against infinitesimal perturbations. Both the laminar (trivial) base flow and some nontrivial turbulent regime can coexist spatially in the interval $\left[R e_{\mathrm{g}}, R e_{\mathrm{c}}\right]$. The flow through a straight pipe of circular section and the plane shear flow between two parallel plates moving in opposite directions (plane Couette flow (PCF)) represent extreme situations since the corresponding laminar base flow profiles are 
linearly stable for all $R e$, i.e. $R e_{\mathrm{c}}=\infty$. An interesting recent review of current issues can be found in Mullin and Kerswell (2005). Here we shall study PCF considered as a paradigmatic case of wall-bounded flow with potential relevance to less academic systems such as channel or boundary-layer flows.

Parameters characterizing the experiment are the distance $2 h$ between the plates driving the flow (wall-normal direction $y$ ) and the in-plane dimensions $L_{x}$ and $L_{z}$ in the streamwise and spanwise directions, respectively. The physical properties of the fluid under shear are all contained in its kinematic viscosity $v$ and the Reynolds number is defined as $R e=U h / v$, where $U$ is the speed of the plates. Laboratory experiments were initially focussed on the determination of $R e_{\mathrm{g}}$ (Bottin 1998, Bottin and Chaté 1998, Bottin et al 1998) in systems of aspect ratio $L_{x, z} / 2 h$ from moderate to large.

Figure 1 (top, left) illustrates turbulence decay observed in experiments where a flow prepared at very high $R e$ is quenched down to some final $R e \lesssim R e_{\mathrm{g}}$, using time series of the turbulent fraction, the relative surface of the region occupied by turbulence. Extensive statistics were gathered on the transients' lifetimes which were found to be roughly exponentially distributed at a given $R e$, with mean lifetimes increasing rapidly as $R e$ approached $R e_{\mathrm{g}} \simeq 325$ from below. Irregular patches with locally oblique shape were observed; see figure 1 (top, right). Later experiments (Prigent 2001, Prigent et al 2003, for a review see Prigent and Dauchot 2005) showed that, at larger aspect ratio, an upper threshold $R e_{\mathrm{t}} \simeq 410$ could be defined, above which turbulence was essentially featureless and below which it was present in the form of alternately laminar and turbulent oblique bands. Upon decreasing $R e$, the upper part of the transitional range $\left[R e_{\mathrm{g}}, R e_{\mathrm{t}}\right]$ was marked by a continuous increase of the oblique modulation of the turbulence intensity (figure 1, centre). In the lower part, the bands were fragmented and less regularly organized (figure 1, bottom). PCF is the zero-curvature limit of counter-rotating cylindrical Couette flow where the oblique bands take the form of spirals (Coles and Van Atta 1967) and have essentially the same characteristics with the differences attributed to the fact that streamwise boundary conditions are periodic in the azimuthal direction. Most of the quantitative results were obtained in the cylindrical case, while band formation was studied in PCF upon decreasing $R e$ by steps, taking snapshots of the pattern, and just measuring wavelengths (Prigent 2001, p 75-76). In particular the decay of bands around $R e_{\mathrm{g}}$, which was not studied in detail, will be our main concern here.

Besides experiments of the type sketched above, many theoretical and numerical studies have been devoted to the decay of turbulence in wall-bounded flows, often using domains of the size of the minimal flow unit (MFU), the smallest domain in which turbulence can be sustained at low to moderate Reynolds number (Jiménez and Moin 1991). MFU-sized domains have periodic boundary conditions in the direction(s) perpendicular to the wall at distances of the order of the relevant wall-normal length scale (the tube's diameter, distance between plates and boundary layer thickness). This approach has shed light on the general mechanisms sustaining the turbulent regime, with streamwise vortices generating streaks by lift-up, and the instability of streaks feeding back the vortices (see Hamilton et al 1995, Waleffe 1997). In the transitional range, the structure of the nontrivial state remains highly coherent at small scale owing to the moderate value of the Reynolds number. The flow regime that develops is then not developed turbulence but a milder chaotic evolution, which is in principle describable in terms of low-dimensional dynamical systems, allowing the use of concepts and tools of deterministic chaos theory. In this context, the relevant objects are unstable periodic orbits that are special exact solutions of the Navier-Stokes equations in the corresponding MFU (Nagata 1990, Waleffe 2003, Toh and Itano 2003, Cvitanović and Gibson 2010, etc) and the homoclinic tangle formed from the invariant manifolds attached to them. Within this framework, the decay of turbulence is described as a chaotic transient 

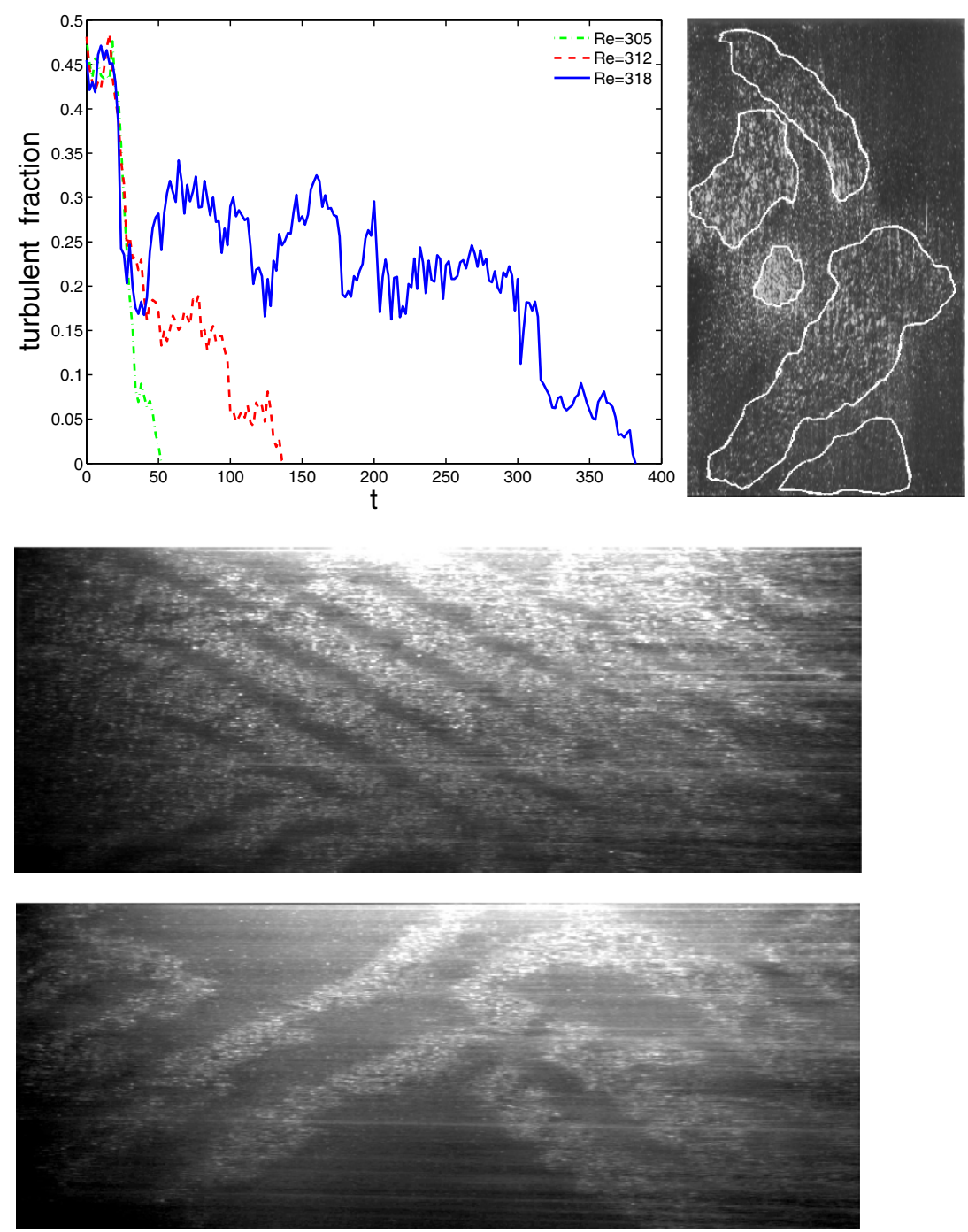

Figure 1. Top: experimental results of Bottin (1998). Left: turbulent fraction as a function of time in experiments where turbulent flow is quenched down to the value indicated in the legend in a system of size $L_{x}=286 \mathrm{~h}, L_{z}=72 \mathrm{~h}, 2 \mathrm{~h}=7 \mathrm{~mm}$; time $t$ is given in seconds. Right: snapshot of a transient solution at $R e=320$ in a wider system with $L_{x}=572 \mathrm{~h}, L_{z}=145 \mathrm{~h}, 2 \mathrm{~h}=3.5 \mathrm{~mm}$, indicating the region detected to be turbulent. The streamwise direction is vertical. The original data and image courtesy of S Bottin. Bottom: Prigent's very large aspect ratio experiment $\left(L_{x}=770 \mathrm{~h}, L_{z}=340 \mathrm{~h}\right.$, gap $\left.2 \mathrm{~h}=1.5 \mathrm{~mm}\right)$. In the middle of the transitional range $(R e=376$, top) a near-ideal pattern of oblique bands is obtained, but closer to $R e_{\mathrm{g}},(R e=331$, bottom), bands break down. The streamwise direction is horizontal. Images courtesy of A Prigent.

for which a lifetime can be defined and the probability distribution of lifetimes is found to be exponentially decreasing (Eckhardt and Faisst 2005, Eckhardt et al 2008, Schneider et al 2010, etc).

Until now, it has however turned out to be difficult to establish a clear correspondence between laboratory experiments and numerical simulations analysed in the dynamical system setting. This is because the spatial coherence introduced by periodic boundary conditions 
at small distances used in simulations supports the reduction to low-dimensional dynamical systems and the temporal chaos interpretation, but stays at odds with the physical situation that corresponds to flow domains extended in one dimension (pipe or duct flow) or two dimensions (PCF, channel and boundary layer flow), situating the transitional problem in a fully spatiotemporal perspective. Puff intermittency in pipe flow (Moxey and Barkley 2010, Barkley 2011) and oblique turbulent patterning in PCF are manifestations of the spatiotemporal processes at work. Like in the theory of critical phenomena in thermodynamic phase transitions (e.g. Stanley 1988), the effective space dimension is expected to play an important role. For example, weakly turbulent, streamwise localized, equilibrium structures with definite global structure called puffs are known to control turbulence decay in the pipeflow case. They do not seem to have any counterpart in PCF where turbulent spots are always found to experience wild streamwise and spanwise shape fluctuations without an apparent overall structure except for a tendency to obliqueness. Also, no equivalent of the puff-slug transition in pipe flow (Duguet et al 2010b, Barkley 2011) has yet been identified in PCF and other two-dimensional (2D) cases.

The oblique band regime in PCF is less well documented than the puff regime in pipe flow. It was numerically studied by Barkley and Tuckerman (2005a,b, 2007) who recovered it by performing simulations in periodic domains elongated in the direction normal to the bands but narrow (a few MFUs) in the complementary in-plane direction. Although this approach gives a reliable account of streamwise correlations essential to the band formation, Philip and Manneville (2011), it has not yet given much information about the decay mechanisms. Others, in particular Duguet et al (2010a), considered periodic extended domains of sizes of the order of Prigent's experimental setup and performed fully resolved numerical simulations in quantitative agreement with the experiments. The corresponding computational load was, however, an obstacle to the consideration of the many configurations relevant to decay. Our recent work, Manneville and Rolland (2010), showed that band formation is a highly robust phenomenon that withstands drastic resolution decrease, reliably reproducing the wavelength variations and qualitatively preserving most of the features of the flow, apart from a general but tolerable downward shift of the transitional range. Here, we take advantage of this result to study domains sufficiently wide to fit several wavelengths of the oblique band pattern in long-lasting simulations but at moderate computational cost, expecting a realistic rendering of the breakdown of bands not targeted before. Section 2 is devoted to the numerical conditions of the experiments, in particular the preparation of initial conditions, and the post-treatment. Section 3 describes our main result that band collapse involves two basic processes, first the formation of a laminar gap breaking a band section 3.2 and next a slow retreat of the band fragments left by the breaking section 3.3. The discussion in section 4 begins with a review of the theoretical concepts introduced to account for the decay of turbulence and next situates our findings within this framework, showing that they give a reasonable understanding of experimental observations.

\section{Preliminaries}

\subsection{Numerical considerations}

We perform direct numerical simulations of the Navier-Stokes equations using Gibson's public domain code CHANNELFLow (Gibson 2010). It implements a pseudo-spectral scheme using Chebyshev polynomials in the wall-normal direction $y$ where no-slip boundary conditions are applied at $y= \pm 1$ in units of $h$, the half-gap between the plates driving the flow. Fourier modes are used to deal with the space dependence in the streamwise $(x)$ and spanwise $(z)$ 
directions with periodic boundary conditions at distances $L_{x}$ and $L_{z}$, again expressed in units of $h$. The speed $U$ of the counter-translating plates serves to define the turn over time $h / U$ as the time unit.

Preliminary work of Manneville and Rolland (2010) has shown that the main features of transitional PCF are appropriately reproduced in under-resolved simulations, with apparently just a downward shift of the Reynolds number range where the bands are observed. As can be anticipated for wall-bounded flows at moderate Reynolds numbers, the most significant effect of resolution lowering lies in the account of the wall-normal dependence hence the number of Chebyshev polynomials used. We showed that this number can be drastically reduced from 27 to 33, which is usually considered as sufficient in the transitional range $R e \sim 300-400$ (Duguet et al 2010a), down to 11 without losing the transitional range in extended geometry. The price to be paid was a lowering of the interval $\left[R e_{\mathrm{g}}, R e_{\mathrm{t}}\right]$ from $[325,410]$ in experiments (Prigent et al 2003) or well-resolved simulations (Duguet et al 2010a) down to [210, 270] for $N_{y}=11$. Increasing $N_{y}$ was shown to improve the situation readily and the value $N_{y}=15$ was indicated as a good choice. On the other hand, in the low- $R e$ range of interest here, the flow is highly coherent and rather smooth at the scale of the MFU, with size $\ell_{x}=12.8 \mathrm{~h}$, $\ell_{z}=4.2 h$ according to Waleffe (2003). Taking effective space steps $\delta x^{\prime}=1.5$ and $\delta z^{\prime}=0.5$ makes about 8.5 points in each direction to describe fluctuations at the MFU scale. After aliasing removal according to the $2 / 3$ rule, this gives $\delta x=1$ and $\delta z=1 / 3$ for the evaluation of the nonlinear terms in the pseudo-spectral scheme. A good compromise between acceptable rendering of the transitional range and moderate computational load at a large aspect ratio was thus suggested to be $N_{y}=15, N_{x}=L_{x}$ and $N_{z}=3 L_{z}$, shifting [Re $e_{\mathrm{g}}, R e_{\mathrm{t}}$ ] down to $[\approx 275, \approx 350]$, below experimental observations by $15 \%$ only, while preserving most of the features of the banded state qualitatively, in particular the wavelength selection properties.

The fact that a lowered resolution renders the transition reasonably well seems due to the fact that the self-sustaining process Waleffe (1997) involves a small number of wall-normal modes. An appropriate account of streamwise correlations in the featureless turbulent regime beyond $R e_{\mathrm{t}}$ is, however, needed in view of a realistic restitution of the bands (Philip and Manneville 2011). This is testified by the failure of the too drastic wall-normal modelling described by Lagha and Manneville (2007a), which happens to be corrected upon a slight improvement as shown by Manneville and Rolland (2010).

Numerical experiments discussed here have been performed with $L_{x}=432$ and $L_{z}=$ 256, which is the largest size that could easily be managed with our desktop computer. Simulation during one time unit $(h / U)$ takes about $1 \mathrm{~min}$. Knowing from experiments that the pattern's wavelengths are $\lambda_{x} \simeq 110 h$ and $85 h$ around $R e_{\mathrm{g}}$ decreasing to about $40 h$ around $R e_{\mathrm{t}}$, the values taken for $L_{x, z}$ lead us to expect a pattern with about three wavelengths close to $R e_{\mathrm{g}}$. It can be argued that, if curvature effects could be neglected, using periodic boundary conditions would make the exactly counter-rotating CCF even closer to our computational implementation than actual PCF. This would not change our expectation much since in the azimuthal $(\theta$, streamwise) and axial ( $z$, spanwise) directions, the wavelengths vary by steps from $\lambda_{\theta}=100 h$ and $\lambda_{z}=40 h$ close to $R e_{\mathrm{t}}$ to $155 \mathrm{~h}$ and $70 \mathrm{~h}$ around $R e_{\mathrm{g}}$, respectively (Prigent et al 2003).

\subsection{Preparation of the initial state}

The main purpose of the paper is to study how turbulent bands decay in a large-aspect-ratio system. The system is thus prepared in the featureless regime at $R e=450$. The Reynolds number is then decreased by steps down to the range of interest around $R e_{\mathrm{g}} \approx 275$. At each step, simulations are performed during limited time lapses. For $R e \geqslant R e_{\mathrm{t}} \approx 350$, the flow is 

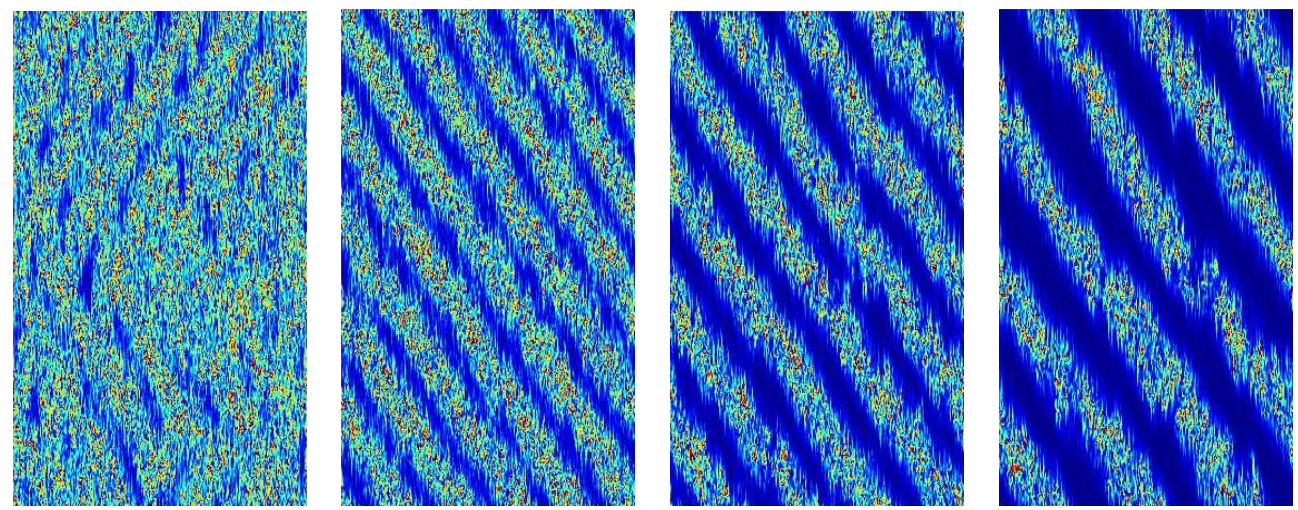

Figure 2. Flow patterns obtained in the simulation at $R e=340, t=20000$ (left), $R e=320$, $t=12000$ (centre left), $R e=290, t=18000$ (centre-right) and $R e=280, t=12000$ (right) represented using $e(x, z, t)$. The streamwise direction is along the vertical axis, here and henceforth. $L_{x}=432, L_{z}=256, N_{x}=432, N_{y}=15, N_{z}=768$. Colour scale from 0 (blue) to 0.1 (red); in each figure $e(x, z, t)$ peaks to above 0.2 , whereas, from left to right, the means vary from 0.0363 to 0.0175 .

still featureless. Figure 2 (left) displays the state obtained close to $R e_{\mathrm{t}}$ at $R e=340$ in the intermittent regime (Prigent et al 2003, Barkley and Tuckerman 2005a,b). Here, and in most of the figures showing patterns, we display the local perturbation energy field averaged over the thickness

$$
e(x, z, t)=\frac{1}{2} \int_{-1}^{+1} \mathrm{~d} y\left[\frac{1}{2} \tilde{\mathbf{v}}^{2}\right],
$$

where $\tilde{\mathbf{v}}=\mathbf{v}-y \hat{\mathbf{x}}, \mathbf{v}$ being the total velocity field and $y \hat{\mathbf{x}}$ the laminar flow. Averaging over the thickness neglects subtleties linked to laminar flow overhanging turbulent flow or the reverse at the laminar-turbulent interface (Coles and Van Atta 1967, Barkley and Tuckerman 2007 ) but this does not change the qualitative conclusions. At $R e=340$, the two orientations allowed by symmetries are represented and the wavelengths corresponding to peaks in the power spectrum of $e(x, z, t)$ are $\lambda_{x}=86.4$ and 108 and $\lambda_{z}=42.7$ and 51.2, in good agreement with values expected close to $R e_{\mathrm{t}}$. Farther away from $R e_{\mathrm{t}}$ at $R e=320$, the pattern is now well organized (figure 2, centre left) and locked on $\lambda_{x}=L_{x} / 5=86.4$ and $\lambda_{z}=L_{z} / 5=51.2$. When $R e$ is further decreased, after a stage of profound reorganisation following Eckhauslike instabilities and the subsequent elimination of dislocations, the system gets locked upon patterns with fewer wavelengths, the first four bands at $R e=290$ (figure 2, centre right) and the next three bands at $R e=280$. Whereas in the latter case the value $\lambda_{z}=85.5$ agrees with experimental findings for PCF, $\lambda_{x}=144$ is somewhat larger than what might be expected but of the same order of magnitude as what is found in CCF. Such slight discrepancies are not surprising in view of the commensurability conditions imposed by the periodic boundary conditions fixing the dimensions of the system and the associated resonant locking of the wavelengths achieved in the pattern. The Reynolds number is then decreased to $R e=275$ for which the three-band pattern obtained seems sustained, as far as one can tell from a simulation of finite duration (here $t_{\max }=25000 \mathrm{~h} / \mathrm{U}$ ).

\subsection{Post-treatment of turbulent-laminar coexistence via filtering and thresholding}

As can be seen in figure 1, the transitional regime is characterized by the coexistence of regions that are alternately turbulent and laminar. The identification of these two possible 
local states is performed by thresholding after filtering. Here the discrimination uses $e(x, z, t)$. We take a Gaussian filter function in the form $G_{\Delta}(r)=\left(6 / \pi \Delta^{2}\right)^{1 / 2} \exp \left(-6 r^{2} / \Delta^{2}\right)$ yielding the transfer function $\hat{G}_{\Delta}(k)=\exp \left(-k^{2} \Delta^{2} / 24\right)$ which has the same second moment as the box filter of width $\Delta$ (Pope 2000, p 561ff.). To take into account the difference in coherence along the streamwise and spanwise directions, we choose an effective filtering box which is rectangular and scales as the size of the domain for which an exact nontrivial solution was found by Waleffe (2003), since such coherent states are expected to be the smallest structures relevant in the low- $R e$ spatiotemporal context we are dealing with. We thus take $\Delta_{x}=12.8 \kappa$ and $\Delta_{z}=4.2 \kappa$, where $\kappa$ is a factor of the order of one to be specified (usually larger than 0.5 and smaller than 4). Several averaged quantities can then be computed, conditioned by the fact that the region is considered either turbulent or laminar, according to whether the local filtered perturbation energy is larger or smaller than some cutoff value $e^{\mathrm{c}}$. The discussion of the filtering and thresholding problems is straightforward.

The mean perturbation energy is defined as

$$
E(t)=\frac{1}{L_{x} L_{z}} \iint \mathrm{d} x \mathrm{~d} z e(x, z, t) .
$$

Let $\mathcal{I}_{\mathrm{t}}(x, z, t)$ be the indicator function of the turbulent region after thresholding. The instantaneous turbulent fraction is then

$$
F_{\mathrm{t}}(t)=\frac{1}{L_{x} L_{z}} \iint \mathrm{d} x \mathrm{~d} z \mathcal{I}_{\mathrm{t}}(x, z, t),
$$

and one can further define a mean turbulent energy, i.e. the mean energy per unit surface in the region identified as turbulent:

$$
E_{\mathrm{t}}(t)=\frac{\iint \mathrm{d} x \mathrm{~d} z \mathcal{I}_{\mathrm{t}}(x, z, t) e(x, z, t)}{\iint \mathrm{d} x \mathrm{~d} z \mathcal{I}_{\mathrm{t}}(x, z, t)} .
$$

The time averages of these quantities $\langle F\rangle,\langle E\rangle$ and $\left\langle E_{\mathrm{t}}\right\rangle$ can then be computed when the system has reached its stationary regime. The effects of changes in $\kappa$ and $e^{\mathrm{c}}$ on the laminar-turbulent detection are illustrated and discussed in figures 3 and 4 . There is no ideal relative filter size $\kappa$ and energy cutoff $e^{\mathrm{c}}$ but once they are fixed, qualitative comparisons between different states are fully informative and quantitative variations of the observables are of significance. In the following, we take $e^{\mathrm{c}}=3 \times 10^{-3}$ and $\kappa=2$ most of the time.

\section{Turbulence decay of PCF in extended geometry}

\subsection{Phenomenology of band decay}

The flow field obtained at $R e=275$ and $t=7000$ is used as an initial condition to study the lower transitional range around $R e_{\mathrm{g}}$. Always starting from this state, several simulations are performed by decreasing $R e$ down to a slightly smaller value of $R e$ and waiting until the laminar regime is reached. Figure 5 displays the variation with time of the distance $D$ to the laminar flow defined as $D=\sqrt{2 E}$ during each experiment.

The first experiment in this series is performed at $R e=273.75$. Each of the three bands forming the initial state breaks in turn but recovers (an example of a broken band is given for $t=3000$ in figure 6, left) until two bands break nearly simultaneously around $t=7000$. Each broken band recedes, yielding a more complex pattern with several band segments separated by a single continuous band. The situation is illustrated at $t=10250$ in the central 

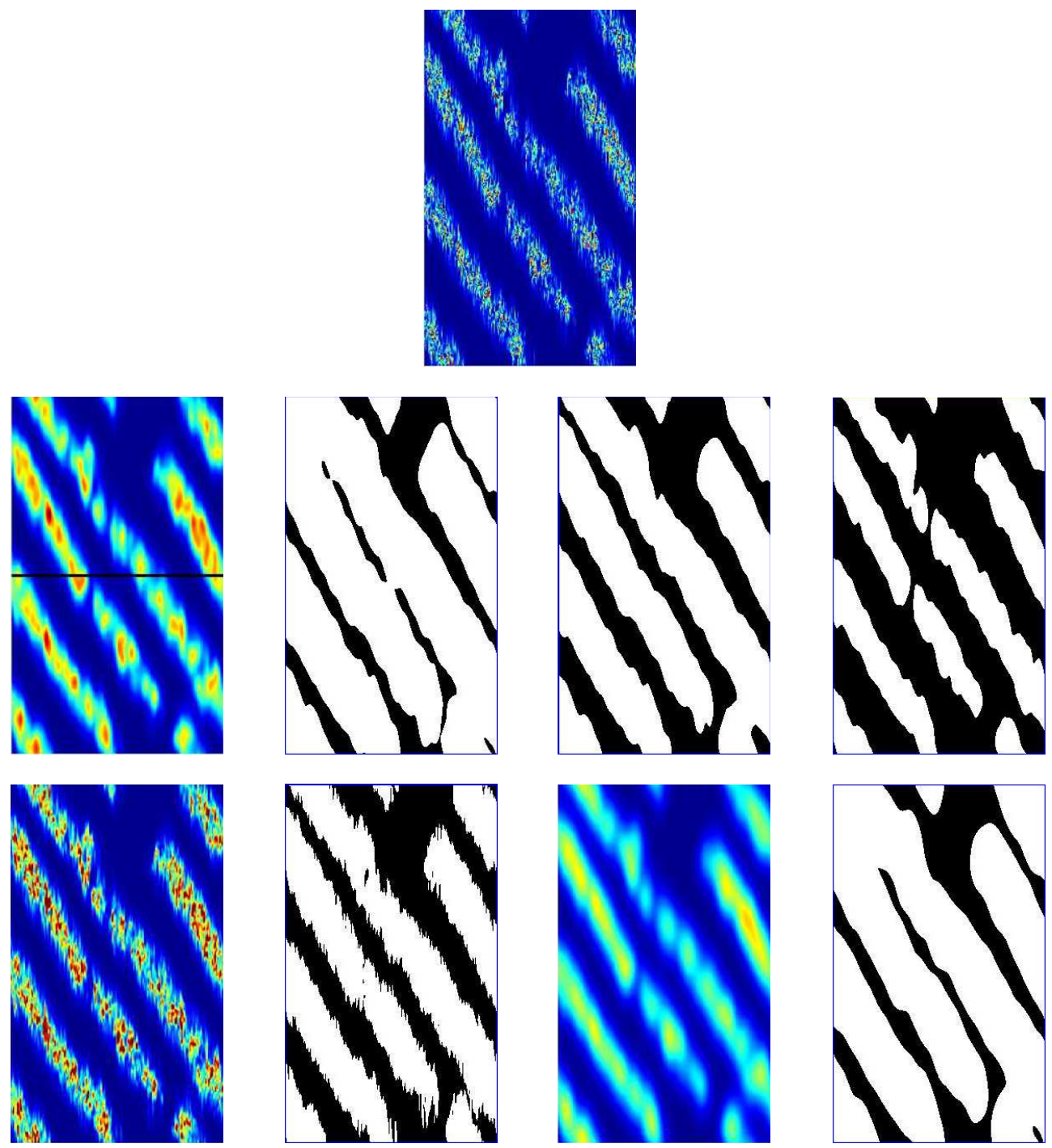

Figure 3. Illustration of filtering and thresholding. Top: flow pattern obtained in the simulation at $R e=271.25, t=1250$. Bottom: filtered state and indicator functions of the turbulent region (white) for several values of $\kappa$ and energy cutoff $e^{\mathrm{c}}$. The first line: $\kappa=2$ and $e^{\mathrm{c}}=0.001,0.003$, 0.010 ; in the leftmost panel the horizontal line indicates the location of the spanwise profiles in figure 4. The second line: $\kappa=0.5$ (left) and $\kappa=4$ (right), in both cases $e^{\mathfrak{c}}=0.003$. Except in the top image where the same colour scale is used as in figure 2, i.e. from 0 to 0.1 with an energy peaking at 0.2450 , here the colour scale ranges from 0 to 0.0555 as fixed by the image with $\kappa=2$ on the left of the first line. The filtered energy peaks at 0.1046 for $\kappa=0.5$ and at 0.0371 for $\kappa=4$.

panel in figure 6. Next, the band portions recede and disappear while the continuous band breaks, retracts along its length (snapshot at $t=15250$ ) and breaks (snapshot at $t=17000$ ). Fragments left then rapidly decay and at $t \approx 19250$ the flow is entirely laminar.

For the second experiment we set $R e=272.5$. Snapshots during decay are displayed in figure 7 . The difference to the previous experiments is that the first band to break does not recover but retracts (see the left snapshot at $t=4500$ ). When it has completely disappeared, we are left with a state displaying two unevenly spaced bands (centre left snapshot at 

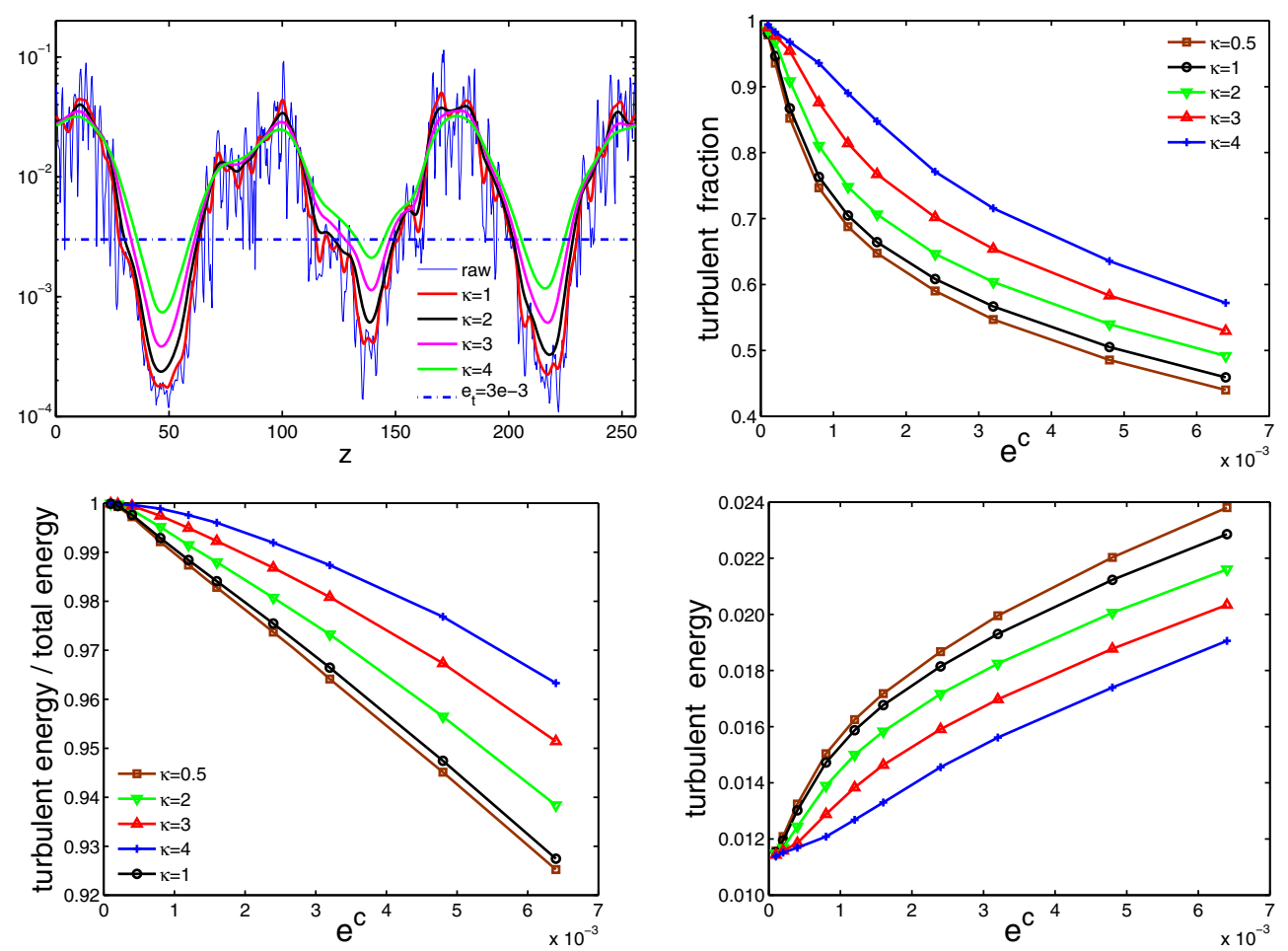

Figure 4. Top left: energy profiles along $z=L_{z} / 2$, raw and filtered with different values of $\kappa$ as indicated, the same state as in figure 3. Filtering is clearly insufficient for $\kappa=1$ and too strong for $\kappa=4 ; \kappa=2$ or 3 seem more satisfactory; in the following we keep $\kappa=2$. Top right: variation of the turbulent fraction as a function of $e^{\mathrm{c}}$ for several values of $\kappa$; as can easily be understood from the images in figure 3 and the profiles in the graphs on the left, the turbulent fraction decreases as $e^{\mathrm{c}}$ increases at given $\kappa$ and increases as $\kappa$ increases at given $e^{\mathrm{c}}$, as a consequence of the nesting of domains with different filter widths and thresholds. Bottom: relative energy content of the turbulent fraction (left) and turbulent energy (right) as functions of $e^{\mathrm{c}}$ for several values of $\kappa$; observed trends derive from the previous observation in a straightforward way. No significant break appears in these curves that would suggest an optimum value to $e^{\mathrm{c}}$. Wanting most of the energy to be concentrated in the domain labelled 'turbulent', we choose $e^{\mathrm{c}}=0.003$ (dash-dotted line in the top-left panel) so that about $97.5 \%$ of the energy is allocated to the turbulent region for $\kappa=2$ in the case considered.

$t=12000)$. This state seems rather robust since the bands alternately break from time to time but recover, while the distance between them tends to equalize. One of the remaining bands breaks at $t \approx 15300$ and then retracts. The last band breaks at $t \approx 20600$ and recedes regularly till the end of the transient.

The third experiment is for $R e=271.25$. Two out of the three bands present in the initial state shortly break, possibly at several places, and each portion retracts as described previously. Figure 8 illustrates the end of the transient. The last band breaks around $t=3500$ (left panel) and all the turbulent patches recede in parallel, disappearing one after the other (other panels). The transient ends at $t \approx 7750$. The last experiment of this series, with $R e=270$, is not illustrated since it displays the same stages as the previous one. The whole relaxation takes a shorter time, the transient ending at $t \approx 5250$.

Before going further, let us illustrate the use of the filtering-and-thresholding procedure introduced in section 2.3. Figure 9 corresponds to the transient at $R e=273.75$ but is typical 


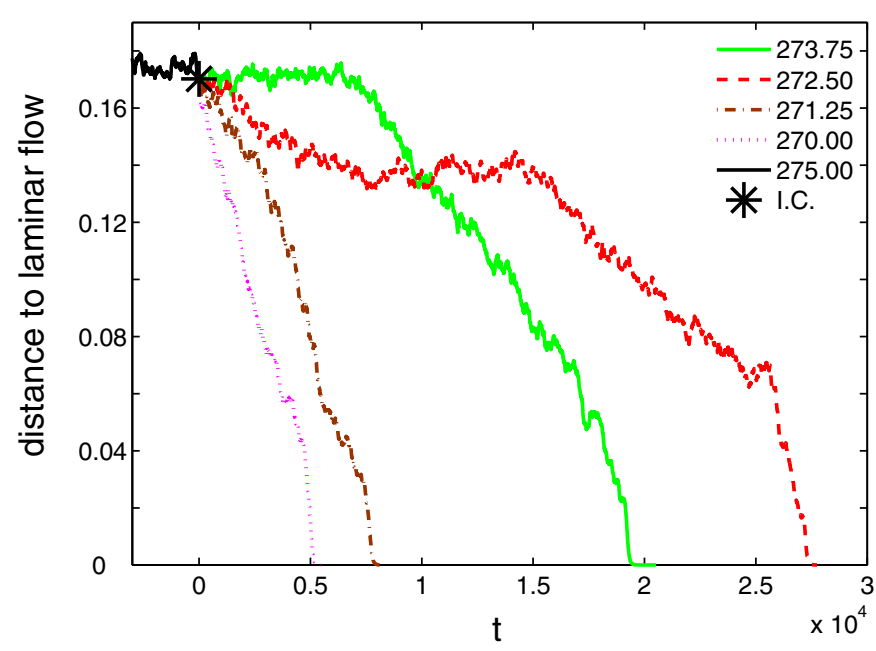

Figure 5. Variation of distance $D$ to the laminar flow as a function of time during decay starting from the three-band state at $R e=275$ at $t=7000$ (asterisk at the end of the continuous black line) for $R e=273.75$ (green, full line), 272.50 (red, dashed), 271.25 (brown, dot-dashed) and 270.00 (magenta, dotted).
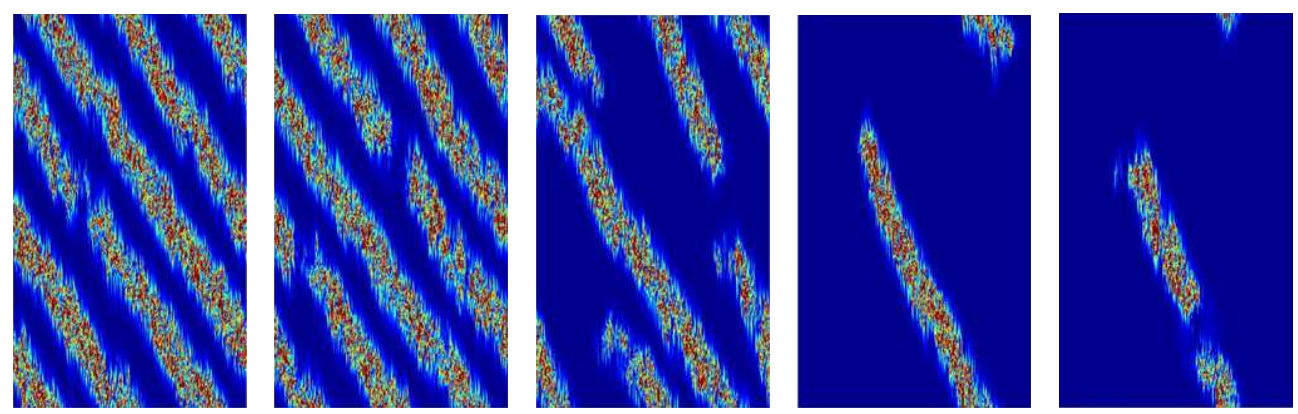

Figure 6. Snapshots taken during the decay at $R e=273.75$ from state at $R e=275, t=7000$. Time is reset at the beginning of the experiment. From left to right: $t=3000$ : a band breaks but recovers; $t=7250$ : two bands break; $t=10250$ : complex intermediate state; $t=15250$ : a single band portion remains; $t \simeq 17000$ : the band segment breaks.

of the other cases studied. It can be seen that up to appropriate rescaling, all along the transient down to its end, the mean perturbation energy closely follows the turbulent fraction (which is expected since the perturbation-energy content of the region identified as laminar is negligible if thresholding is properly performed). The continuous-band metastable state observed till $t \approx 7000$ is clearly visible. On the other hand, the mean turbulent energy and the local-energy peak value do not show any change except at the very end of the transient where they abruptly fall to zero. This confirms the relevance of our previous findings based on a different procedure for the identification of the laminar and turbulent local states Rolland and Manneville (2011a) when applied to decaying turbulence.

As seen in figures 6-8, the bands seem to keep a constant width all along their decay. Two basic processes are at work: first, a large-scale collapse of a band in its bulk over a region of length comparable to its width and, next, a slow and regular retreat due to small-scale collapse of the turbulent state at the laminar-turbulent interface. If the laminar gap appearing 

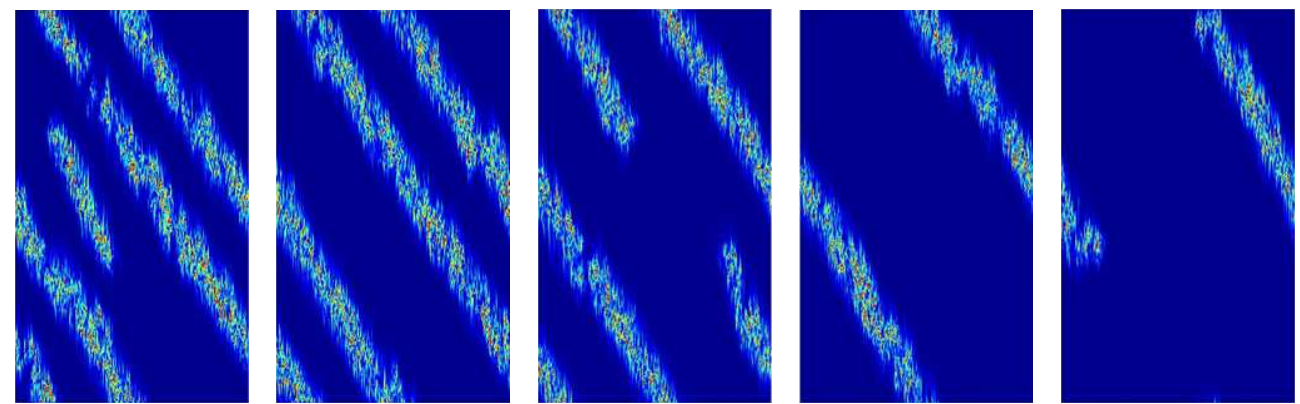

Figure 7. Snapshots taken during the decay at $R e=272.5$. From left to right: at $t=4500$ some time after the breakdown of the first band; at $t=12000$ with two unevenly spaced ban ds; at $t=16500$ after breakdown of the second band; at $t=20250$ with one continuous band remaining; at $t=24000$ during the decay of the last band.
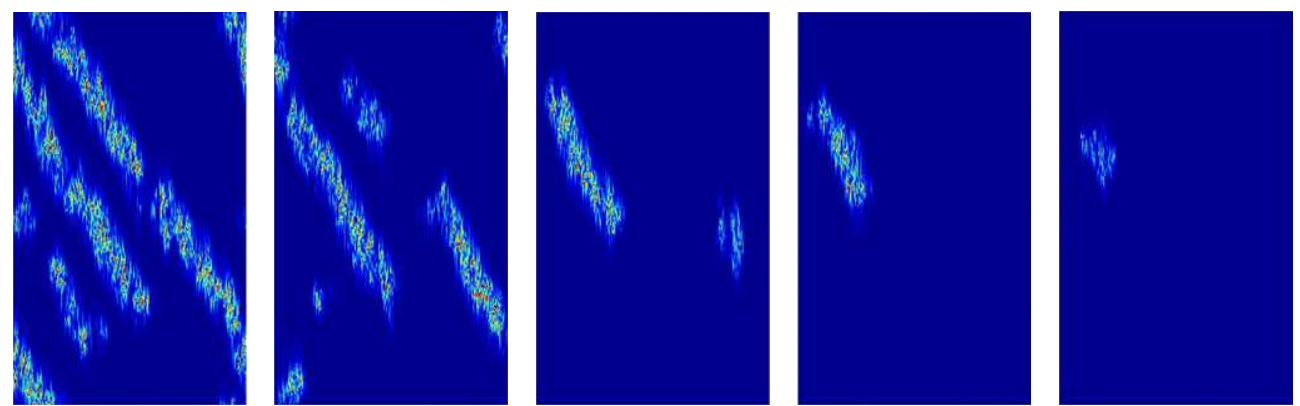

Figure 8. Snapshots taken during the decay at $R e=271.25$. Time is reset at the beginning of the experiment. From left to right: $t=3500,4500,5500,6500$ and 7500 .

in the band is not large enough, the band can recover its continuous character. In this respect, the continuous bands can be viewed as metastable against the nucleation of a sufficiently large laminar gap, recovery corresponding to germs smaller than critical. This point of view was put forward by Pomeau in chapter 4 of Bergé et al (1998) in the general context of transitional PCF. It is here adapted to the band regime that was not yet uncovered at that time. Its relevance is particularly clear at $R e=273.75$ where the system waits during $7000 \mathrm{~h} / \mathrm{U}$ before decaying after several unsuccessful attempts, and at $R e=272.50$ where the two-band state remains in place during a long time interval with similar aborted events.

After a sufficiently wide gap has been nucleated, the steady retraction of the turbulent domains is then easily understood as a propagation problem for a stable state (laminar) that gains over a metastable one (turbulent). Except when $R e$ is low enough and band breaking becomes a competitive process, the band portions issued from a breaking stay in a single piece and the evolution is essentially a trimming process at both ends. Owing to the stochastic character of the turbulent state, and the elongated shape of the receding turbulent domains, this second stage then fits the framework put forward by Pomeau (1986) of a contamination process akin to 1D directed percolation Hinrichsen (2000): below the percolation threshold, the front separating the absorbing state (laminar) and the active state (turbulent) advances at a statistically constant velocity, so as to make each turbulent band portion retract. A similar but opposite situation is studied by Duguet and Schlatter (2011) who considers the growth of the turbulent state above the percolation threshold in a predominantly $1 \mathrm{D}$ geometry elongated in the spanwise direction. 


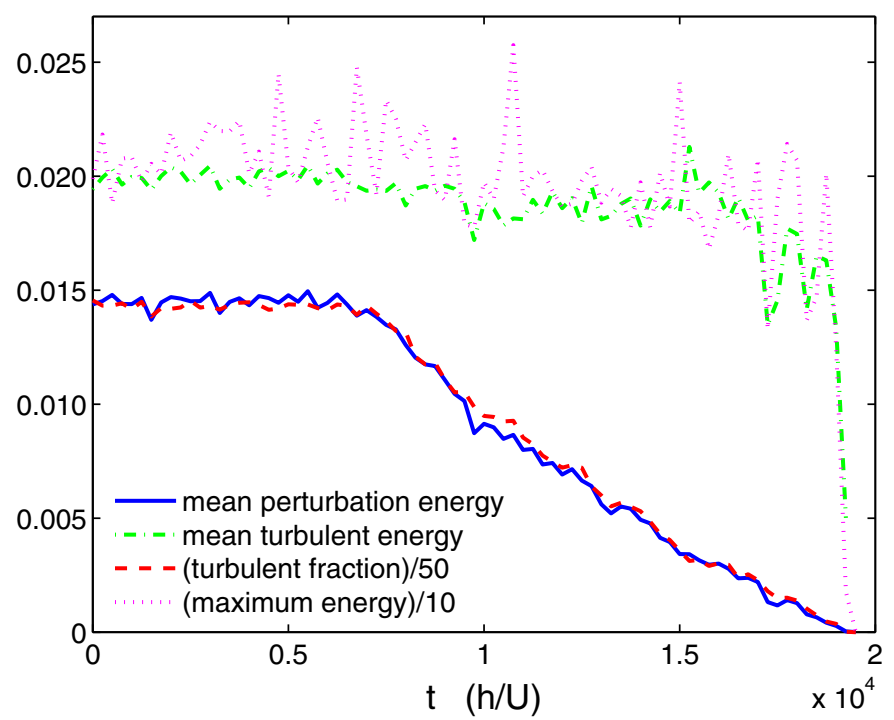

Figure 9. Statistical characterization of the bands during the transient at $R e=273.75$ (figures 5 and 6) after filtering with $\kappa=2$ and thresholding with $e^{\mathrm{c}}=0.003$.

We conjecture that the two processes are different manifestations of the same physics which originates from the chaotic character of the dynamics at the scale of the MFU at values of the Reynolds number for which chaotic transients are expected. As such, the involved probabilities should critically depend on $R e$. We substantiate this viewpoint by examining these two processes more quantitatively in the next subsections.

\subsection{Nucleation of a laminar gap}

Following Prigent et al (2003), in our previous work (Rolland and Manneville 2011a,b) we analysed the transitional range as a pattern formation problem, using a Langevin-Landau model governing an order parameter submitted to a strong background noise induced by turbulence. In particular, we studied orientation changes when bands are well formed in terms of a stochastic process with two wells separated by a barrier, each well corresponding to one of the two possible orientations. Changes were then understood as resulting from large deviations due to the cumulative effects of noise. The problem of band breakdown can be set in a similar framework and seen as a random exit problem (van Kampen 1983), i.e. when the trajectory in state space reaches a boundary beyond which the system tumbles in a new state. In the present context, as it fluctuates a germ (laminar gap) can grow to some critical size beyond which it is bound to expand indefinitely (turbulent band recedes), while, as long as the boundary is not crossed, the turbulent band recovers (the germ fluctuates back), so that the system has just experienced a large excursion towards the laminar state (aborted event).

Since it has been observed above that the minimum size of a laminar gap leading to the breakdown of a band is of the order of the width of the turbulent band itself, we can try to relate the nucleation problem to the collapse of turbulence in domains of sizes $L_{x} \times L_{z}$ of the order of the size of the elementary pattern cell $\lambda_{x} \times \lambda_{z}$. Afterwards, we can consider the whole band as formed from a series of such elementary cells, making the supplementary assumption that these cells are independent so that the collapse probabilities simply add. Strictly speaking, this assumption is certainly wrong owing to long-range interactions induced by large-scale 

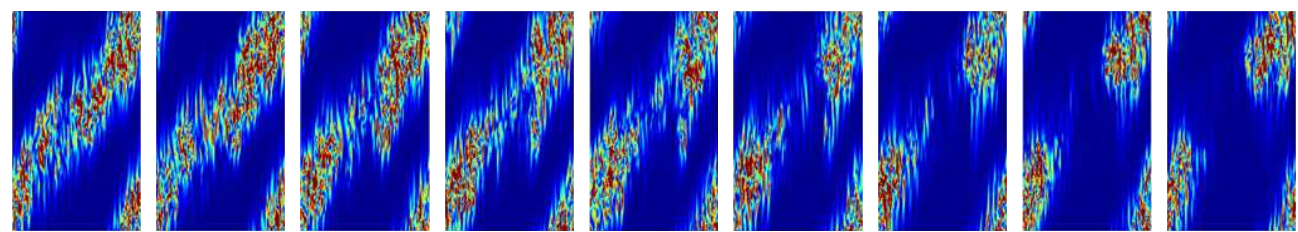

Figure 10. Snapshots taken during the fatal opening of a laminar gap in the band for the small domain $(144 \times 84)$ at $R e=271.25$. From left to right, time increases by steps of $50 \mathrm{~h} / \mathrm{U}$ from $t=2700 \mathrm{on}$. Remember the periodic boundary conditions.

flows outside the turbulent bands (Barkley and Tuckerman 2007) but it can serve as a guide. Accordingly we study excursions away from the oblique-band turbulent state in systems of sizes of the order of $\lambda_{x} \times \lambda_{z}$, hence accommodating a single band, see figure 10, the leftmost image. We mainly consider $L_{x}^{\text {ss }}=144$ and $L_{z}^{\text {ss }}=84$, where the superscript 'ss' means a 'small system'. This corresponds to $\lambda_{x}$ and $\lambda_{z}$ exactly one-third of $L_{x}$ and $L_{z}$, as obtained in our main simulations. Computations are performed at the same local resolution as for the large system, i.e. $N_{x}^{\mathrm{ss}}=L_{x}^{\mathrm{ss}}$ and $N_{z}^{\mathrm{ss}}=3 L_{z}^{\mathrm{ss}}$. Figure 10 displays the opening of the laminar gap in the band for $R e=271.25$ in such a domain. After a few aborted events, this opening is fatal to the continuity of the band and the transient terminates about $1600 h / U$ later. In line with the basic assumption of a cumulative effect of local noise driving the excursions away from the turbulent state, we base our study on the distance $D$ to the laminar state. In a large deviation perspective, this quantity is roughly proportional to the amount of local turbulence at the origin of the stochastic dynamics and therefore appropriately related to the fluctuating position of the laminar-turbulent interface in an additive way. The changes in the statistics of the time series of $D$ as a function of $R e$ are thus our primary matter of interest.

Figure 11 (top) displays the time series of $D$ for $R e=275$ over a very long time lapse $\left(3 \times 10^{5} h / U\right)$. As far as one can tell from one numerical experiment, this specific turbulent regime is considered as sustained. Excursions such as the one at $t \approx 8500$ are neither sufficiently dangerous nor sufficiently frequent to make the system decay to laminar flow. By contrast, for $R e<275$, decay is observed in finite duration simulations, owing to the more frequent occurrence of more dangerous excursions. As can be appreciated from figure 11 (bottom), whatever the value of $R e$, decay is seen to follow similar paths and the stage of irreversible relaxation to laminar flow takes place as soon as $D$ is smaller than about 0.03 . A statistically robust trend is, however, already observed when $t$ is about $1500 \mathrm{~h} / \mathrm{U}$ before the end of the transient (arbitrarily fixed when $D$ reaches $10^{-5}$ ), which corresponds to the opening time of the fatal laminar gap; see figure 10 and related text. The instantaneous value of $D$ then begins to depart from its mean as computed by discarding the last $2000 \mathrm{~h} / \mathrm{U}$ before decay. The slow decrease that follows the opening corresponds to a regular retreat of the remaining turbulent band fragment very much like in the wide system. Further examination of the data suggests that, for $R e$ about 275 , this happens systematically when $D$ crosses $\approx 0.13$ from above and that, when this is the case, the system does not return to the oblique-banded turbulent state and is bound to decay within the next $1500 \mathrm{~h} / \mathrm{U}$.

Probability density functions (PDF) of the instantaneous value of $D$ are then constructed. They are displayed in figure 12 for different values of $R e$. In this figure one can see that the most probable value slowly decreases with $R e$ but more importantly that the probability of low values, corresponding to the dangerous excursions, increases drastically in a narrow range of $R e$. Decay has not been observed for $R e \geqslant 275$ in any experiment of duration $\leqslant 3 \times 10^{5} \mathrm{~h} / U$. Extrapolation of the exponentially decreasing tail in the corresponding PDF, 

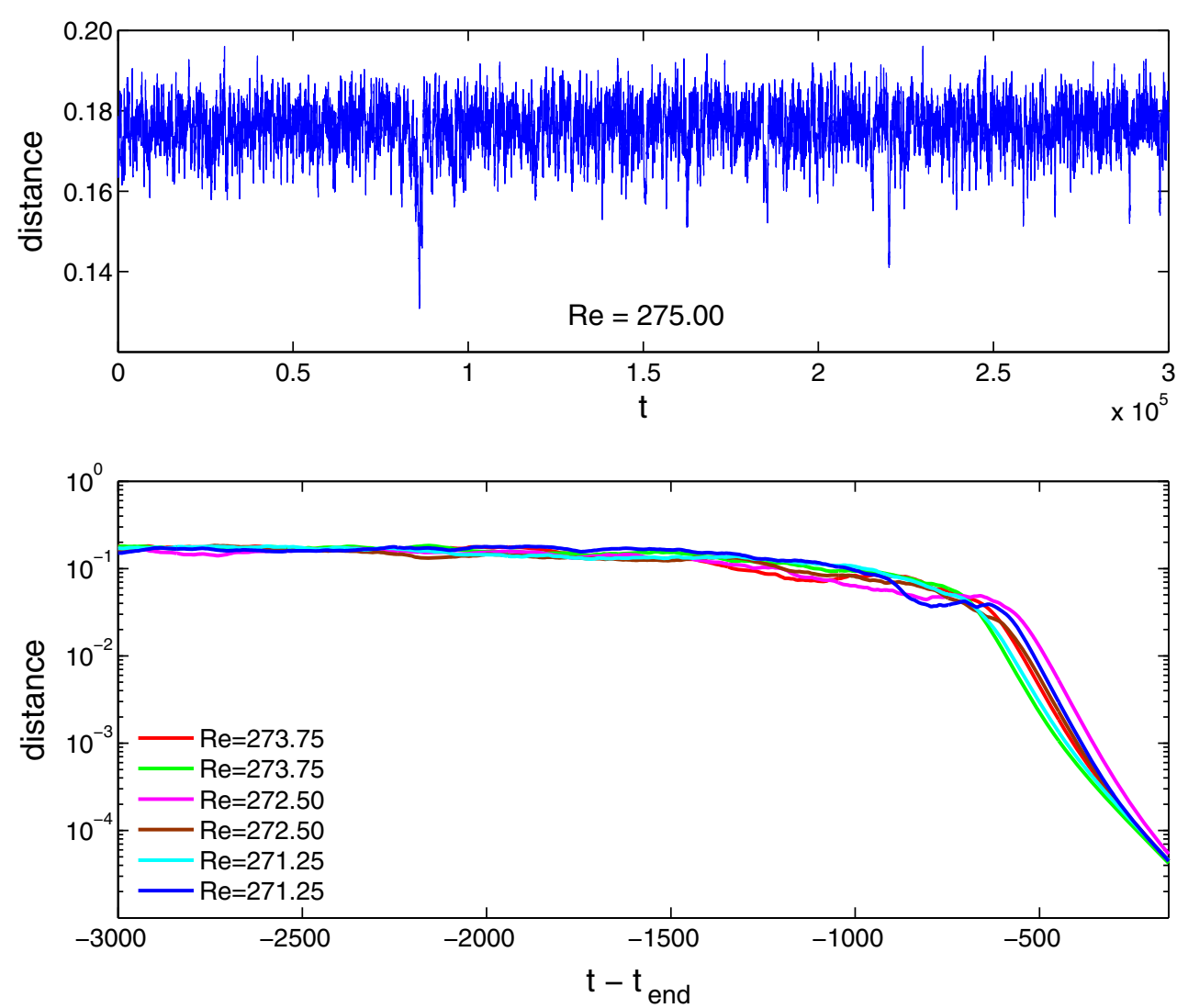

Figure 11. Time series of the distance to laminar flow $D$. Top: for $R e=275$. Bottom: during the last decay stage for several transients and various $R e<275$; the origin of times, $t=0$, is set when $D=10^{-5}$.

however, suggests that this event has a very low but finite probability of occurrence, i.e. could only be observable in simulations much longer than $10^{5}$. For the transient cases, the statistics rests only on simulations longer than $6000 \mathrm{~h} / U$ and on the parts of the time series excluding the 2000 last time units, cumulating at least $10^{5}$ measures per value of $R e$, thus requiring the study of several independent transients (up to about ten for $R e=271.25$ ).

It does not seem necessary to go beyond this hand-waving approach to the band breaking process because, although the trends shown are certainly real and qualitatively correct, the results are quantitatively sensitive to the limited set of trajectories that are followed and not necessarily representative of a genuine ensemble average. As already mentioned, the resolution of the simulation is also at the origin of the general downward shift of the transitional range that limits extrapolation to the fully resolved case. The size of the pattern's elementary cell also plays a role: a similar study has been conducted with $L_{x}^{\mathrm{ss}}=108$ and $L_{z}^{\mathrm{ss}}=64$, one-fourth of the big system's dimensions, thus keeping the same angle but corresponding to a smaller wavelength. Results, not shown, are quantitatively similar, but with a slight downward shift of the corresponding range of $R e$. Decay turns out to be more difficult (less probable) in the $108 \times 64$ domain than in the $144 \times 84$ domain, which can be understood as due to stronger constraints brought by the periodic boundary conditions at a smaller distance, yielding narrower laminar stripes between the turbulent bands and discouraging the 


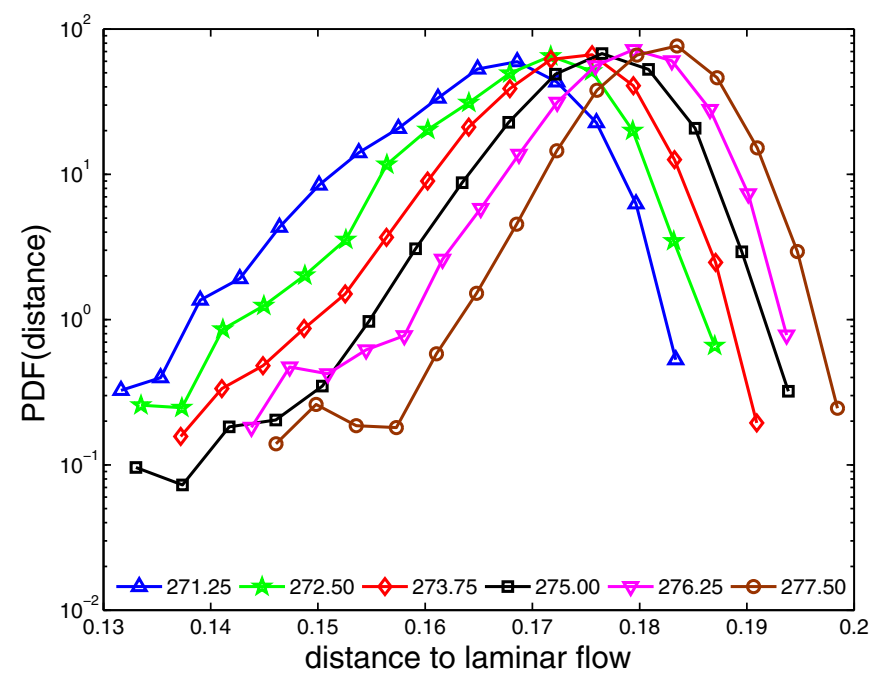

Figure 12. Probability distribution functions of the fluctuations of the distance to the laminar state for different values of $R e$; see text for details.

formation of the large laminar patches associated with the dangerous excursions of $D$ toward low values, in agreement with similar observations made in small systems designed to check the Ginzburg-Landau approach (Rolland and Manneville 2011a).

Note, however that, still in the large deviation perspective, consideration of figure 12 offers an explanation for the observed exponential decrease of transient lifetime distributions that is an alternative to that provided by the chaotic transient paradigm (Eckhardt et al 2008). Whereas the latter is undoubtedly appropriate for systems at the MFU scale, i.e. confined, it fails to account for spatiotemporal features inherent in extended systems of sizes sufficient to accommodate turbulence modulation, i.e. $\approx \lambda_{x} \times \lambda_{z}$ or greater. Indeed, upon assuming that the trajectory followed by the system at given $R e$ samples the PDF of $D$ at random and that a return to laminar flow inevitably takes place within a short time when $D$ happens to reach a value below some cut-off (here $\approx 0.13$ ), decay can then be seen as a Poisson process controlled by the corresponding small probability (hence exponential lifetime distributions when an ensemble of transients are considered), which rapidly decreases as $R e$ increases (hence a rapid increase of the mean lifetime). This explanation ties up with that of Goldenfeld et al (2010) about decaying puffs in pipe flow.

\subsection{Withdrawal velocity}

The second process involved in the decay is a statistically steady retreat of segments issued from the breaking of the continuous bands. These segments get shorter and shorter but keep roughly constant widths, except in the very last stage where they collapse and finally relax under the dominant effect of viscous dissipation as shown in figure 8. The turbulent fraction, or equivalently the mean energy $E$ since the thresholding procedure collects the essentials of the perturbation energy (figure 9), are thus indirect measures of their cumulated length. An average withdrawal speed can be determined from the time variation of the distance to the laminar flow $D=\sqrt{2 E}$. Figure 13 displays part of the data already presented in figure 5 but, in addition, fits the variations of $D$ against affine laws during specific evolution stages. Examining the transients one by one (figures 6-8), it can be seen that, during each of these 


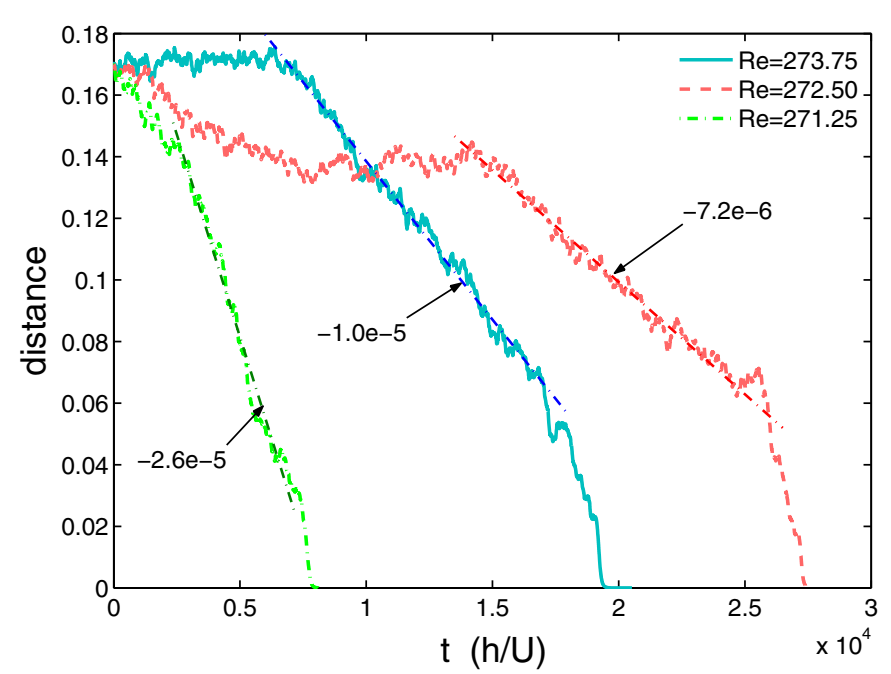

Figure 13. Distance $D$ to laminar flow as a function of time during decay for $R e=273.75,272.50$ and 271.25. Specific evolution stages correspond to steady retreat of the turbulent band segments at speeds that can be estimated from linear fits (dash-dotted lines).

stages, the connectedness of the solution does not change much, with essentially the same number of band fragments. It suggests also that the largest contribution to the evolution comes from the most active parts of a band segment, namely its ends. In the spirit of a directed percolation process, we can then think that the withdrawal at a given end depends on the decay probability of local streaky structures, which at this stage is a weakly varying function of $R e$ in the vicinity of $R e_{\mathrm{g}}$ that we are considering. The global withdrawal speed as determined from the variations of $D$ can then be related to the number of segments involved. This immediately explains why the decay observed for $R e=273.75$, with several bands receding simultaneously (figure 6), is faster than the decay at $R e=272.50$, and also why, in this last case, no slope change is detectable when the third band breaks $(t \sim 20600)$ since, at a given time, only one band is receding, hence only two active ends (figure 7).

Like in the previous subsection, it is, however, neither necessary nor useful to go beyond this qualitative approach. Firstly, as above, limitations due to under-resolution impede reliable predictions for the fully resolved and the experimental cases. Secondly and more importantly, it seems extremely difficult to relate the global withdrawal speed to the local mechanisms operating in the 'active' regions and, in particular, to extract probabilities relevant to the decay of the band segments. In this respect, the situation is less favourable than in the case considered by Duguet and Schlatter (2011) who work with a streamwise-narrow domain. The reason is that the general topology of the solution at a given time, and in particular its connectedness, plays a crucial role. A continuous turbulent band can be prolonged to infinity using the periodic boundary conditions, is effectively endless and has properties different from those of a very long band segment: we have good evidence that, at $R e=275$, solutions formed with continuous bands are stable — or at least prone to band breaking with such a low probability that it could not be observed during simulations of duration much longer than $25000 \mathrm{~h} / \mathrm{U}$, while a band segment at the same $R e$ does recede, as illustrated in figure 14 . The initial condition corresponding to this case is the state obtained at $R e=272.5$ after the breaking of the last band at $t=24000$ (figure 7, right). This initial condition is reproduced in figure 14 (top-left) after re-centering by taking advantage of the periodic boundary conditions. The 

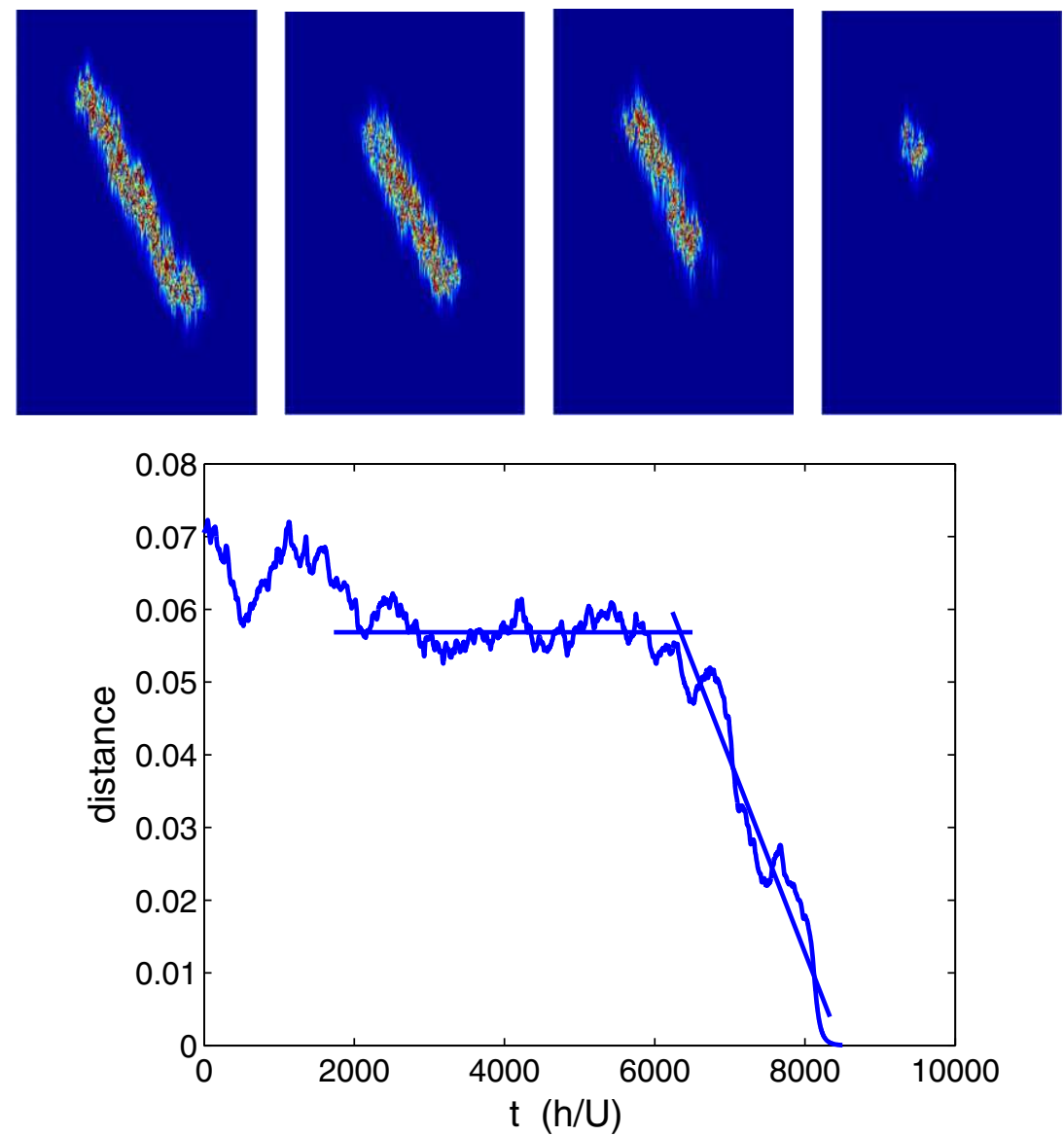

Figure 14. Decay of a band-like but topologically disconnected solution at $R e=275$. From left to right: $t=0,2500,6000$ and 7500. Bottom: distance to laminar flow during the transient.

other images have also been re-centred by the same amount. The graph in figure 14 (bottom) displays the variation of $D$ during this transient, with a plateau from $t \sim 2500$ (second image) to $t \sim 6000$ (third image) preceding a fast collapse ending by the final viscous decay stage.

This observation suggests that the local interpretation of turbulence dynamics in the vicinity of $R e_{\mathrm{g}}$ sketched above has certainly to be corrected from non-local effects: large-scale recirculations with well-defined wall-normal structure are known to exist around turbulent bands (Coles and Van Atta 1967, Barkley and Tuckerman 2007) as well as around turbulent spots (Lundbladh and Johansson 1991, Lagha and Manneville 2007b). These recirculations do not average to zero over the gap so that $v_{x, z}^{\text {mean }}(x, z, t)=\frac{1}{2} \int_{-1}^{+1} \mathrm{~d} y v_{x, z}(x, y, z, t)$ is a good signature of their presence. Figure 15 (right) displays the streamlines of this in-plane average flow. A mild filtering (parameter $\kappa=1$ ) has been performed, which has some smoothing effect only on the flow lines inside the turbulent region and not on its periphery. The right panel of figure 15 shows that this flow stays directed parallel to the turbulent segment in its central portion, much like along the continuous band in the left panel, while near the ends of the segment, it adopts a configuration reminiscent of what is observed for turbulent spots surrounded by a quadrupolar large-scale flow entering streamwise at their tips and getting out spanwise on their sides (Lundbladh and Johansson 1991, Lagha and Manneville 2007b). 

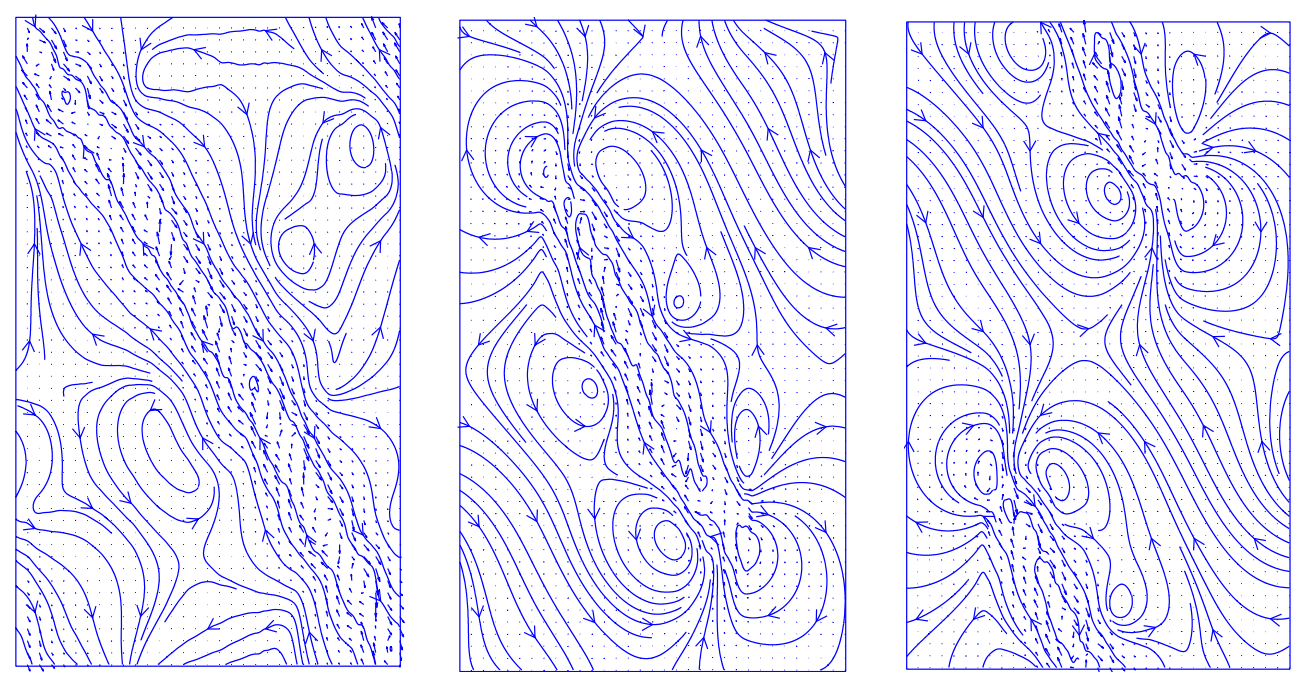

Figure 15. Streamlines of the mean in-plane flow. Left: connected band state obtained during the transient at $R e=272.5$ and $t=20250$ (figure 7, centre-right, re-centred). Right: state shown in figure 14 (left) issued from the breaking of the band and used as an initial condition for the experiment at $R e=275$, centred on the turbulent part (left) and on the laminar gap (right). Notice that, though seemingly present everywhere, the mean flow has small intensity away from the turbulent region, as understood when considering the length of velocity vectors.

Until now, in line with the Langevin perspective introduced by Prigent et al (2003), band breaking has been interpreted as the result of a large deviation linked to the accumulation of individual stochastic processes generated by the chaotic dynamics at the scale of the MFU. However, a full understanding of the formation of a sizeable laminar gap at the origin of band breaking has to take into account the topological reorganization of the large-scale flow, which involves times scales longer than those of the small-scale processes and may not work as the simple random addition underlying the large-deviation concept. This necessary reorganization may contribute to the barrier, ensuring the metastability of the bands at a given $R e$ : longlived banded states stabilized by topological constraints can be observed while band segments generically retract around $R e_{\mathrm{g}}$. Once well understood, processes at the origin of band breaking will also explain the breaking of long turbulent segments into smaller ones since the nucleation of a laminar gap in most parts of a segment excepting its extremities appears similar to the nucleation of a gap at any location of a continuous band (figure 6, the rightmost image): local processes should be identical while the mean flow looks much alike along turbulent band portions of sufficient length, as seen in figure 15 (left and centre left).

We have also observed that band fragments retreat not only at $R e=275$ as mentioned above, but also for $R e>275$. The same initial condition as for the experiment depicted in figure 14 indeed also withdraws for $R e=276.25$ and 277.50 , whereas a band fragment issued from the latter experiment at $R e=277.50$ placed at $R e=278.75$ stays alive for more than $40000 \mathrm{~h} / \mathrm{U}$ (simulation still running) with wild length fluctuations. Reconnection of the large-scale flow in the laminar region between turbulent fragments as depicted in figure 15 (right) may be taken as responsible for the obstruction to the growth of turbulence and the reconstitution of bands expected as $R e$ increases above 275 .

To summarize this section devoted to observations, let us stress that the main process driving the decay of bands is the opening of a laminar gap resulting from the collapse of turbulence over a region much larger than the MFU, likely the output of large deviations of 
an underlying stochastic process, followed by the retreat of turbulent band fragments, but that non-local effects linked to the presence of large-scale flow components have to be taken into account.

\section{Discussion}

In what precedes, we have presented results on the decay of turbulence in plane Couette flow using simulations of the Navier-Stokes equations with mild under-resolution. Before examining the shortcomings of this work and the perspectives it opens, let us expand our brief introduction to the problem given in section 1 and come back to the context of turbulence decay in wall-bounded flows that generically experience a direct transition to turbulence.

Most of the quantitative results obtained in recent years relate to pipe flow and its transition involving puffs. A major finding has been the exponential decay of lifetime distribution of transients, a behaviour interpreted as due to the complicated dynamics trajectories around a homoclinic tangle structuring the phase space around special unstable nontrivial solutions to the Navier-Stokes equations away from the laminar base flow. Even when not stated explicitly, this perspective has implicitly been developed within the framework of (low-dimensional) deterministic dynamical systems, permitted by the coherence of flow fields at the moderate values of $R e$ relevant to the transitional range. The standard approach to chaotic transients then explains the exponentially decreasing variation of transient lifetime distributions, not as the result of some memoryless stochastic process as is often said, but as the result of the sensitivity to initial conditions of trajectories in phase space typical of deterministic chaos. The variation of lifetime distributions then follows from the (indefinite memory of the) unknown positions of initial states taken at random in the domain controlled by the homoclinic tangle and from the fractal structure of the attraction basin of the laminar state that it generates. A good understanding of the nature of transient chaos and the origin of exponentially decaying distributions can be obtained by considering the Hénon model $\left(x_{n+1}=1+y_{n}-a x_{n}^{2}, y_{n+1}=b x_{n}\right)$ at $a=1.3, b=0.3$ for which sustained chaos present at $a=1.4$ decays into a period-7 cycle (Manneville 1982). Transients obtained in flows at the MFU scale are just associated with a dynamics that is less straightforwardly accessible.

The existence of chaotic transients, which fit the temporal framework of low-dimensional dynamical systems, is fundamental to the relevance of the spatiotemporal intermittency (STI) concept to transitional PCF put forward by Pomeau (1986). STI is presented as generic transition scenario in spatially extended systems experiencing a subcritical bifurcation (Manneville 2005). In Pomeau's views, local transient temporal chaos can be converted into sustained spatiotemporal chaos by spatial interactions. The system as a whole, here PCF at a large aspect ratio, is then considered as an assembly of coupled units at the nodes of a lattice. Each unit can be in one of two states, either active or quiescent. The quiescent state, said to be absorbing, is linearly stable, whereas the active state has a finite probability per unit time to decay (Poisson-like process). For PCF, this is precisely the case of the trivial laminar state and the nontrivial chaotic state at the MFU level when $R e$ is sufficiently large. A finite perturbation is necessary to kick a unit out of the absorbing state. Such a perturbation can come from the coupling to a neighbouring unit in the active state, which thus contaminates the initially quiescent unit. A straightforward connection to directed percolation was then proposed. This process is a standard stochastic model for flow in porous media, epidemics or forest fires. As such, it can be studied as a critical phenomenon in statistical physics (Stanley 1988) with its possible universality content in mind (Hinrichsen 2000).

In a deterministic context, probabilities introduced in the picture could in principle be related to decay probabilities to quiescent state for the local units, here the MFUs, next 
appropriately coupled to neighbours. This conceptual process has thus received a concrete implementation as coupled map lattices displaying STI. In the systems considered by Chaté and Manneville (1988a), finite lifetime local chaotic transients were converted into sustained spatiotemporal chaos. In this high-dimensional perspective, the decay of transients receives an interpretation that is radically different from the one that prevails in low-dimensional deterministic dynamical systems. Either by changing the rate of local decay or by changing the coupling strength, one can control the transition from a regime where spatiotemporal chaos (turbulence) is sustained to a regime that decays by creating domains of units in quiescent state statistically growing to drive the whole system in the absorbing (laminar) state by making the turbulent domain steadily recede. In contrast, a localized chaotic germ may contaminate the whole system and drive it into sustained spatiotemporal chaos. A threshold separating the two regimes exists and is well defined only at the so-called thermodynamic (large-size) limit, whereas in finite systems of moderate size the transition always keeps probabilistic traits: beyond threshold, the system has a finite but exponentially small probability to decay and, on the other hand, germs evolve into the spatiotemporal chaotic regime with probability less than one. Playing with the local dynamics we were able to obtain either continuous, second-orderlike, transitions with critical exponents or discontinuous, first-order like transitions (Chaté and Manneville 1988b, see also, Bohr et al 2001). The latter case was found to be most appropriate to mimic the decay of PCF from turbulence, as shown a decade later by Bottin and Chaté (1998).

This theoretical approach is, however, limited in that it bears no real connection to fluid mechanics, since it rests on strictly analogical modelling and ad hoc choices for the local dynamics and the coupling. To circumvent this limitation, Lagha and Manneville (2007a) developed a more realistic approach based on a severely truncated Galerkin expansion of the Navier-Stokes equations, yielding a set of three coupled partial differential equations governing the in-plane $(x, z)$ dependance of the turbulent fluctuations. The so-derived model reproduced the dominant features of the transition to/from turbulence in PCF at a semiquantitative level, containing the most important hydrodynamic couplings and in particular mean flow corrections to laminar profile at lowest order (Lagha and Manneville 2007a,b). It also served us to test the concept of discontinuous STI transition to account for turbulence decay (Manneville 2009), but unfortunately failed to display the turbulent bands, just showing unorganized laminar troughs in the upper transitional range and no clearly defined upper threshold $R e_{\mathrm{t}}$.

This failure was attributed to the insufficient effective wall-normal $(y)$ resolution rendered by the expansion onto just the first three lowest-order basis polynomials. In order to test this explanation, instead of improving this resolution by increasing the order of the Galerkin basis and getting a much more cumbersome and opaque model, we chose to go back to DNS of the Navier-Stokes equations and to study the effect of a controlled lowering of the resolution (Manneville and Rolland 2010), which led us to suggest the approach as a systematic and consistent modelling strategy, with the set $\left(N_{y}=15, N_{x}=L_{x}, N_{z}=3 L_{z}\right)$ as a good compromise between realism and computational load.

The picture emerging from our observations is thus rather different from what derives from the low-dimensional analysis of the dynamics at the MFU scale and the homoclinic tangle paradigm. Complexity at this level, possibly involving chaotic transients, is not enough and correlations at larger scale have to be accounted for. Recent work by Philip and Manneville (2011) showed that patterns appear only in large enough periodic domains able to afford modulations of the intensity of the self-sustaining process over streamwise distances of order at least $70 \mathrm{~h}$. By the same token, this helped us to understand the success of the approach initiated by Barkley and Tuckerman (2005a,b, 2007), who were able to reproduce 
the bands in narrow domains at an angle with the streamwise direction: thanks to periodic but shifted boundary conditions at MFU distances, this mimics what happens in wider domains. A detailed understanding of turbulence decay in systems of concrete interest has thus to include spatiotemporal effects that can only be accounted for by considering sufficiently wide domains, either in both in-plane directions or elongated but appropriately oriented.

In this context, our study of band decay clearly points out a two-stage process. Firstly, when bands are continuous, they can break with some probability function of $R e$. This breaking takes place when a small set of neighbouring strongly correlated MFUs in the chaotic state collapse, thus making an indentation in the laminar-turbulent interface. This indentation can increase and form a small gap in the turbulent band. At this stage, the gap can either enlarge or close back. This evolution is probabilistic, owing to underlying chaotic dynamics. Large deviations in the properties of this stochastic process are suggested to explain the formation of a laminar gap (figure 12) as discussed by Rolland and Manneville (2011b) for orientation fluctuations. A similar point of view was developed by Goldenfeld et al (2010) to explain the statistics of decaying puffs in pipe flow. Secondly, when the laminar gap is sufficiently large, the same stochastic process can trim the so-formed turbulent band fragment at its ends, making it shorter and shorter, regularly but still only in the long term and at a statistical level, in the spirit of STI (Pomeau 1986, Bottin and Chate 1998). However, to improve the relevance of this approach to fully hydrodynamic conditions, corrections associated with nonlocal couplings via large-scale flows will have to be introduced (figure 15).

To close this discussion, let us remark that despite different protocols, different aspect ratios, different boundary conditions and the expected $R e$-shift due to under-resolution in the numerics, experimental results shown in figure 1 (left) are strikingly similar to our findings (e.g. figure 14). The average turbulence intensity indeed exhibits long periods of fluctuations at roughly constant turbulent fraction, interrupted by marked drops corresponding to the breakdown of turbulence over sizeable regions and a final viscous decay stage. In this figure, time is given in seconds and the fluid is water with kinematic viscosity $v \approx 10^{-6} \mathrm{~m}^{2} \mathrm{~s}^{-1}$. With $h=3.5 \mathrm{~mm}$ and $R e=325 \sim R e_{\mathrm{g}}$, for the time unit one gets $h / U=h^{2} /(\nu \operatorname{Re}) \sim 0.04 \mathrm{~s}$, so that the longest transient shown in the figure is about $9000 h / U$, of the same order of magnitude as ours in similar conditions.

\section{Conclusion}

Our recourse to under-resolved simulations allowed the study of many different cases in wide domains during long durations at moderate computational cost. The previously obtained validation of under-resolution as a modelling strategy (Manneville and Rolland 2010) gives us good reasons to believe that the results described here closely shadow a situation taking place in the fully resolved case (and in the experiments) at slightly larger values of $R e$. The genericity and robustness of the emerging picture suggest that processes uncovered during band decay will remain relevant up to a mere adjustment of probabilities as functions of $R e$. The combination of band breaking and subsequent turbulence retreat results in a large variety of breakdown scenarios, and in a whole distribution of lifetimes that would demand huge numerical resources to be studied statistically. Via nucleation theory, the putative largedeviation origin of the phenomenon, however, proposes an interpretation of exponential decay time distributions by events taking place in physical space (spatiotemporal viewpoint) rather than in phase space as implied in the current chaotic transient paradigm (temporal viewpoint). We believe that, for large aspect ratio systems of interest in experiments, the former is more credible than the latter, at least taken alone and not integrated into a broader spatiotemporal framework since chaotic dynamics at the MFU scale is certainly an essential source of 
randomness. However, not only probabilities but also ingredients, local and nonlocal, to the growth/breakdown mechanisms of streaks and vortical structures at the laminar-turbulent spot or band boundaries should receive attention, in particular those involving large-scale mean flows, possibly by extending the modelling approach developed in Lagha and Manneville (2007a,b) to better render the wall-normal dependence and reproduce the bands. All this of course demands confirmation at full resolution using adapted computing power, as well as dedicated experiments. Finally, beyond the specific case of PCF, conclusions would have much interest in other less academic cases of wall-bounded flows, channels and boundary layers.

\section{Acknowledgments}

Help with the local numerical implementation of CHANNELFLow from $\mathbf{J}$ Rolland and discussions with D Barkley, F Daviaud, Y Duguet, J Philip, A Prigent, J Rolland and L S Tuckerman are gratefully acknowledged. Thanks are also due to $\mathrm{G}$ Kawahara for his warm welcome in Osaka at an early stage of this work and his continued interest in it.

\section{References}

Barkley D and Tuckerman L S 2005a Turbulent-laminar patterns in plane Couette flow Laminar-Turbulent Transition and Finite Amplitude Solutions (Fluid Mechanics and its Applications vol 77) ed T Mullin and R R Kerswell (Dordrecht: Springer) pp 107-27

Barkley D and Tuckerman L S 2005b Computational study of turbulent laminar patterns in Couette flow Phys. Rev. Lett. 94014502

Barkley D and Tuckerman L S 2007 Mean flow of turbulent laminar pattern in Couette flow J. Fluid Mech. 574 109-37

Barkley D 2011 Simplifying the complexity of pipe flow Phys. Rev. E 84016309

Bohr T, van Hecke M, Mikkelsen M and Ipsen M 2001 Breakdown of universality in transitions to spatiotemporal chaos Phys. Rev. Lett. 86 5482-5

Bergé P, Pomeau Y and Vidal C 1998 L'espace Chaotique (Paris: Hermann)

Bottin S 1998 Structures Cohérentes et Transition vers la Turbulence par Intermittence Spatio-temporelle dans l'Écoulement de Couette Plan PhD Thesis Université Paris-Sud, Orsay, http://tel.archives-ouvertes.fr/tel00001111/en/

Bottin S and Chaté H 1998 Statistical analysis of the transition to turbulence in plane Couette flow Eur. Phys. J. B $6143-55$

Bottin S, Daviaud F, Manneville P and Dauchot O 1998 Discontinuous transition to spatiotemporal intermittency in plane Couette flow Europhys. Lett. 43 171-6

Chaté H and Manneville P 1988a Spatiotemporal intermittency in coupled map lattices Physica D 32 409-22

Chaté H and Manneville P 1988b Continuous and discontinuous transition to spatiotemporal intermittency in twodimensional coupled map lattices Europhys. Lett. 6 591-5

Cvitanović P and Gibson J F 2010 Geometry of the turbulence in wall-bounded shear flows: periodic orbits Phys. Scr. T142 014007

Coles D and Van Atta C W 1967 Digital experiment in spiral turbulence Phys. Fluids Suppl. 10 S120-1

Duguet Y, Schlatter P and Henningson D S 2010a Formation of turbulent patterns near the onset of transition in plane Couette flow J. Fluid. Mech. 650 119-29

Duguet Y, Willis A P and Kerswell R R 2010b Slug genesis in cylindrical pipe flow J. Fluid. Mech. 663 180-208

Duguet Y and Schlatter P 2011 Stochastic motion of a laminar/turbulent interface in a shear flow 13th European Turbulence Conf. (Warsaw, Poland) pp 12-15

Eckhardt B and Faisst H 2005 Dynamical systems and the transition to turbulence Laminar-Turbulent Transition and Finite Amplitude Solutions (Fluid Mechanics and its Applications vol 77) ed T Mullin and R R Kerswell (Dordrecht: Springer) pp 35-50

Eckhardt B, Faisst H, Schmiegel A and Schneider T M 2008 Dynamical systems and the transition to turbulence in linearly stable shear flows Phil. Trans. R. Soc. A 366 1297-315 
Gibson J F 2010 Channelflow: a spectral NavierStokes simulator in C++ Technical Report Georgia Institute of Technology, http://www.channelflow.org/

Goldenfeld N, Guttenberg N and Gioia G 2010 Extreme fluctuations and the finite lifetime of the turbulent state Phys. Rev. E 81035304

Hamilton J M, Kim J and Waleffe F 1995 Regeneration mechanisms of near-wall turbulence structures J. Fluid Mech. $287317-48$

Hinrichsen H 2000 Non-equilibrium critical phenomena and phase transitions into absorbing states Adv. Phys. $49815-958$

Jiménez J and Moin P 1991 The minimal flow unit in near-wall turbulence J. Fluid Mech. 225 213-40

Lagha M and Manneville P 2007a Modeling transitional plane Couette flow Eur. Phys. J. B 58 $433-47$

Lagha M and Manneville P 2007b Modeling transitional plane Couette flow. Large scale flow around turbulent spots Phys. Fluids 19094105

Lundbladh A and Johansson A V 1991 Direct simulations of turbulent spots in plane Couette flow J. Fluid Mech 229 499-516

Manneville P 1982 On the statistics of turbulent transients in dissipative dynamical systems Phys. Lett. A 90 327-8

Manneville P 2005 Modeling the direct transition to turbulence Laminar-Turbulent Transition and Finite Amplitude Solutions (Fluid Mechanics and its Applications vol 77) ed T Mullin and R R Kerswell (Dordrecht: Springer) pp 1-33

Manneville P 2009 Spatiotemporal perspective on the decay of turbulence in wall-bounded flows Phys. Rev. E 79025301

Manneville P 2009 Spatiotemporal perspective on the decay of turbulence in wall-bounded flows Phys. Rev. E 79039904 (Erratum)

Manneville P and Rolland J 2010 On modelling transitional turbulent flows using under-resolved direct numerical simulations Theor. Comput. Fluid Dyn. doi:10.1007/s00162-010-0215-5

Moxey D and Barkley D 2010 Distinct large-scale turbulent-laminar states in transitional pipe flow Proc. Natl Acad. Sci. USA 107 8091-6

Mullin T and Kerswell R R (ed) 2005 Laminar-Turbulent Transition and Finite Amplitude Solutions (Fluid Mechanics and its Applications vol 77) (Dordrecht: Springer)

Nagata M 1990 Three-dimensional finite-amplitude solutions in plane Couette flow: bifurcation from infinity J. Fluid Mech. 217 519-27

Philip J and Manneville P 2011 From temporal to spatiotemporal dynamics in transitional plane Couette flow Phys. Rev. E 83036308

Pomeau Y 1986 Front motion, metastability and subcritical bifurcations in hydrodynamics Physica D 23 3-11

Pope S B 2000 Turbulent Flows (Cambridge: Cambridge University Press)

Prigent A 2001 La Spirale Turbulente: Motif de Grande Longueur d'Onde dans les Écoulements Cisaillés Turbulents PhD Thesis Université Paris-Sud, Orsay, http://tel.archives-ouvertes.fr/tel-00261190/en/

Prigent A, Grégoire G, Chaté H and Dauchot O 2003 Long-wavelength modulation of turbulent shear flow Physica D 174 100-13

Prigent A and Dauchot O 2005 Transition to versus from turbulence in subcritical Couette flows Laminar-Turbulent Transition and Finite Amplitude Solutions (Fluid Mechanics and its Applications vol 77) ed T Mullin and R R Kerswell (Dordrecht: Springer) pp 195-219

Rolland J and Manneville P 2011a Ginzburg-Landau description of laminar-turbulent oblique band formation in transitional plane Couette flow Eur. Phys. J. B 80 529-44

Rolland J and Manneville P 2011b Pattern fluctuations in transitional plane Couette Flow J. Stat. Phys. 142 577-91

Schneider T M, De Lillo F, Buehrle J, Eckhardt B, Dörnemann T, Dörnemann K and Freisleben B 2010 Transient turbulence in plane Couette flow Phys. Rev. E 81015301

Stanley H E 1988 Introduction to Phase Transitions and Critical Phenomena (Oxford: Oxford University Press)

Toh S and Itano T 2003 A periodic-like solution in channel flow J. Fluid Mech. 481 67-76

van Kampen N G 1983 Stochastic Processes in Physics and Chemistry (Amsterdam: North-Holland)

Waleffe F 1997 On a self-sustaining process in shear flows Phys. Fluids 9 883-900

Waleffe F 2003 Homotopy of exact coherent structures in plane shear flows Phys. Fluids 15 1517-34 